LBL-37373

UC-413

\title{
Phase Coexistence in Multifragmentation?
}

L.G. Moretto, L. Phair, R. Ghetti, K. Tso, N. Colonna, W. Skulski, and G.J. Wozniak

\author{
Nuclear Science Division \\ Lawrence Berkeley Laboratory \\ University of California \\ Berkeley, California 94720
}

D.R. Bowman, N. Carlin, M. Chartier, C.K. Gelbke, W.G. Gong,

W.C. Hsi, Y.D. Kim, M.A. Lisa, W.G. Lynch, G.F. Peaslee, C. Schwarz,

R.T. de Souza, M.B. Tsang, and F. Zhu

National Superconducting Cyclotron Laboratory and

Department of Physics and Astronomy

Michigan State University

East Lansing, MI 48824

April 1995

This work was supported by the Director, Office of Energy Research, Office of High Energy and Nuclear Physics, Nuclear Physics Division, of the U.S. Department of Energy under Contract No. DE-AC0376SF00098. 


\section{DISCLAIMER}

This report was prepared as an account of work sponsored by an agency of the United States Government. Neither the United States Government nor any agency thereof, nor any of their employees, make any warranty, express or implied, or assumes any legal liability or responsibility for the accuracy, completeness, or usefulness of any information, apparatus, product, or process disclosed, or represents that its use would not infringe privately owned rights. Reference herein to any specific commercial product, process, or service by trade name, trademark, manufacturer, or otherwise does not necessarily constitute or imply its endorsement, recommendation, or favoring by the United States Government or any agency thereof. The views and opinions of authors expressed herein do not necessarily state or reflect those of the United States Government or any agency thereof. 


\section{DISCLAIMER}

Portions of this document may be illegible in electronic image products. Images are produced from the best available original document. 


\title{
PHASE COEXISTENCE IN MULTIFRAGMENTATION?
}

\author{
L.G. Moretto, L. Phair, R. Ghetti, K. Tso, N. Colonna, W. Skulski, and G.J. Wozniak \\ Nuclear Science Division, Lawrence Berkeley Laboratory \\ Berkeley, California 94720 USA \\ and
}
D.R. Bowman ${ }^{3}$, N. Carlin ${ }^{4}$, M. Chartier, C.K. Gelbke, W.G. Gong, W.C. Hsi, Y.D. Kim, M.A. Lisa, W.G. Lynch, G.F. Peaslee ${ }^{8}$, C. Schwarz, R.T. de Souza, 9 M.B. Tsang, and F. Zhu ${ }^{11}$ National Superconducting Cyclotron Laboratory and Department of Physics and Astronomy Michigan State University, East Lansing, MI 48824 USA

\begin{abstract}
The charge $(Z)$ distributions from intermediate energy heavy-ion reactions depend upon the multiplicity $n$ of intermediate mass fragments through a factor of the form $e^{-c n Z}$. Experimentally $c$ starts from zero at low values of the transverse energy $E_{t}$ and reaches a saturation value at high $E_{t}$. In a liquid-gas phase diagram, $c=0$ for the saturated vapor, while $c>0$ for the unsaturated vapor. It is suggested that in the $c \approx 0$ regime the source evaporates down to a sizable remnant, while for $c>0$ the source vaporizes completely. Percolation of a finite system portrays a behavior similar to that observed experimentally.
\end{abstract}

\section{Introduction}

Multifragmentation has been frequently associated with the liquid-vapor phase transition. A large amount of theoretical work ${ }^{1,2,3,4,5,6,7}$ describes multifragmentation as due either to droplet formation near criticality (critical opalescence), or to the appearance of both liquid and vapor phases after crossing the spinodal line. Percolation theories have been developed to mimic critical behavior in finite systems 8,9. Behavior near criticality has been inferred from the experimentally observed power-law dependence of the intermediate mass fragment (IMF, $3 \leq Z \leq 20$ ) charge distribution 10,11,12,13. More recently, critical exponents have been extracted from the dependence of the moments of the charge distribution upon quantities, like the

1Present address: INFN, Via Amendola 173, 70126 Bari, Italy

${ }^{2}$ Present address: Heavy Ion Laboratory, Warsaw University, PL 02097, Poland

${ }^{3}$ Present address: Chalk River Laboratories, Chalk River, Ontario K0J 1J0, Canada

${ }^{4}$ Present address: Instituto de Fisica, Universidade de Sao Paulo, C.P. 20516, CEP 01498, Sao Paulo, Brazil

${ }^{5}$ Present address: GANIL, BP 5027, F-14021 Caen, France

${ }^{6}$ Present address: Lawrence Berkeley Laboratory, Berkeley, CA 94720

${ }^{7}$ Present address: National Laboratory for High Energy Physics, 1-1 Oho, Tsukuba, Ibaraki 305, Japan

${ }^{8}$ Present address: Physics Department, Hope College, Holland, MI 49423

${ }^{9}$ Present address: Gesselschaft fur Schwerionenforschung, D-6100 Darmstadt, Germany

10 Present address: Department of Chemistry, Indiana University, Bloomington IN 47405

11 Present address: Brookhaven National Laboratory, Upton, NY 19973 


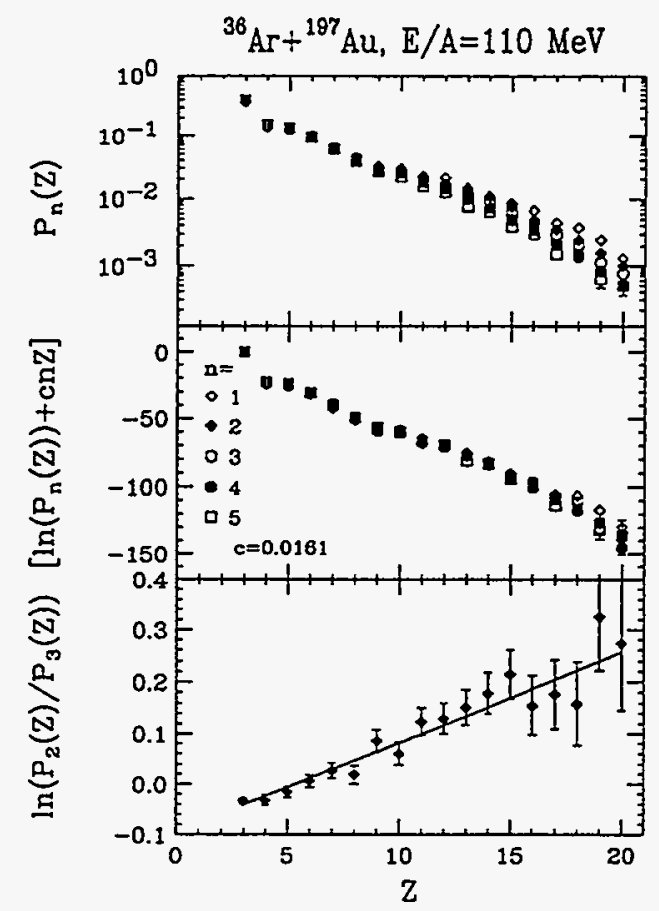

Fig. 1. Top panel: the $n$ gated charge distributions $P_{n}(Z)$ for the reaction ${ }^{36} \mathrm{Ar}+{ }^{197} \mathrm{Au}$ at $E / A=110$ $\mathrm{MeV}$. The charge distributions were constructed from events with $E_{t}=650 \pm 20 \mathrm{MeV}$ and $n=1-5$. Middle panel: the "reduced" charge distribution ${ }^{17}$ for the same data using the indicated value of $c$. (The data here are normalized at $Z=3$ ). Bottom panel: the $\log$ of the ratio of $P_{2}(Z) / P_{3}(Z)$. The slope corresponds to $c$ for $n=2$ (see Eq.(4)). The statistical error bars are shown for errors larger than the symbol size.

charge multiplicity, that are typically associated with temperature and/or excitation energy ${ }^{14}$.

In this light, one of the goals of multifragmentation studies is the mapping of the nuclear phase diagram, in particular of the boundaries of the phase coexistence region. In this paper we report evidence for a possible transition from a two-phase to a one-phase regime obtained from the analysis of charge distributions and their dependence upon IMF multiplicity.

\section{Reducible and Thermal Charge Distributions}

It has been observed ${ }^{15,16}$ that, for a large number of reactions, the IMF multiplicities are binomially distributed, and that the probability $P_{n}$ to emit $n$ IMFs can be reduced to a one-fragment emission probability $p$. This is true at all transverse energies $\left(E_{t}=\sum_{i} E_{i} \sin ^{2} \theta_{i}\right.$ where the sum is over all particles in an event and $E_{i}$ and $\theta_{i}$ are the kinetic energy and polar angle of particle $i$, respectively) ${ }^{15,16}$. The resulting one-fragment emission probability appears to have a Boltzmann form $p=e^{-B / T}$, assuming that the temperature $T \propto \sqrt{E_{t}}$ and $B$ represents an average barrier for fragment emission. The thermal nature of $p$ was inferred from the observation of 
linear Arrhenius plots $\left(\ln p \propto 1 / \sqrt{E_{t}}\right)$ where $E_{t}$ is assumed to be proportional to excitation energy.

The reducibility of $P_{n}$ to $p$ was verified empirically for fragments with $Z \geq 3$. The implications of such reducibility for the charge distributions as a function of the fragment multiplicity $n$ were discussed in ref. ${ }^{17}$. In this work it was found that the experimental charge distributions for any value of $n$ and $E_{t}$ could be expressed by the equation:

$$
P_{n}(Z) \propto \exp -\left[\frac{-F(Z)}{\sqrt{E_{t}}}+n c Z\right]
$$

where $F(Z)$ represents a universal function of $Z$.

The dependence of $P_{n}(Z)$ on $n$ is illustrated in Fig. 1 for the reaction ${ }^{36} \mathrm{Ar}+{ }^{197} \mathrm{Au}$ at $E / A=110 \mathrm{MeV}$. In the top panel, the different symbols show the charge distributions for different fragment multiplicities $n$ at a fixed $E_{t}=650 \mathrm{MeV}$. The slight differences in the $n$ gated charge distributions nearly disappear when plotted in the "reduced" form $F(Z)$ shown in the middle panel.

Again, assuming $E_{t}$ proportional to the excitation energy of the source, Eq. (1) can be written in terms of the temperature $T$ :

$$
P_{n}(Z) \propto \exp -\left[\frac{B(Z)}{T}+n c Z\right]=\exp -\left[\frac{\Delta E(Z)}{T}+\Delta S(n, Z)\right]
$$

The first term in the exponent was interpreted ${ }^{17}$ as an energy or enthalpy term, associated with the energy (enthalpy) needed to form a fragment. The second term was claimed to point to an asymptotic entropy associated with the combinatorial structure of multifragmentation. It was observed that a term of this form arises naturally in the charge distribution obtained by the least biased breaking of an integer $Z_{0}$ into $n$ fragments. Such a $Z$ distribution is given approximately by:

$$
P(Z)=\frac{n^{2}}{Z_{0}} e^{-\frac{n Z}{Z_{0}}}=c n^{2} e^{-c n Z} .
$$

While this form obviously implies charge conservation, it is not necessary that charge conservation be implemented as suggested by Eq. (3). In fact it is easy to envisage a regime where the quantity $c$ should be zero. Sequential thermal emission is a case in point. Since any fragment does not know how many other fragments will follow its emission, its charge distribution can not reflect charge conservation under the constraint of $n$ fragments.

On the other hand, in a simultaneous emission controlled by a $n$-fragment transition state ${ }^{18,19}$, fragments would be strongly aware of each other, and would reflect such an awareness through the charge distribution.

\section{Phase Coexistence(?)}

The question then arises whether $c=0$ or $c>0$, or even better, whether one 


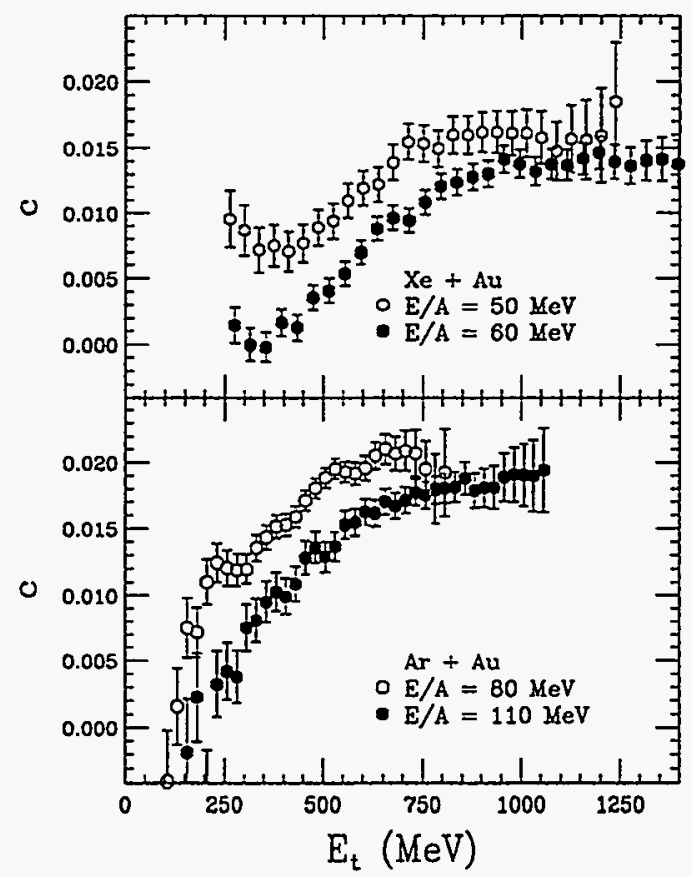

Fig. 2. Plots of the coefficient $c$ versus $E_{t}$ for the reactions ${ }^{129} \mathrm{Xe}+{ }^{197} \mathrm{Au}$ at $E / A=50$ and $60 \mathrm{MeV}$ (top panel) and ${ }^{36} \mathrm{Ar}+{ }^{197} \mathrm{Au}$ at $E / A=80$ and $110 \mathrm{MeV}$ (bottom panel). The error bars are statistical.

can identify a transition from a regime for which $c=0$ to a new regime for which $c>0$. In order to answer this question, we have studied the charge distributions as a function of fragment multiplicity $n$ and transverse energy $E_{t}$ for a number of systems and excitation energies. Specifically, we will present data for the reaction ${ }^{36} \mathrm{Ar}+{ }^{197} \mathrm{Au}$ at $E / A=80$ and $110 \mathrm{MeV}$ and the reaction ${ }^{129} \mathrm{Xe}+{ }^{197} \mathrm{Au}$ at $E / A=50$ and $60 \mathrm{MeV}$.

Several approaches were used to extract $c$ from the charge distributions. If the charge distributions are exponential (as is sometimes the case, $P_{n}(Z) \propto e^{-\alpha_{n} Z}$ ), it is sufficient to extract from them the exponential coefficient $\alpha_{n}$. From the $n$ dependence of $\alpha_{n}$, the quantity $c$ is readily extracted ${ }^{17}$. A more general approach which does not depend on any specific form for the charge distribution is to construct at each $E_{t}$ the ratio

$$
\frac{P_{n}(Z)}{P_{n+1}(Z)}=e^{c Z}
$$

A value of $c$ can be extracted for each $n$ by taking the log of this ratio and finding the slope of the resulting graph (see bottom panel of Fig. 1). A weighted average (over all IMF multiplicities $n$ ) for $c$ can then be constructed at all $E_{t}$. Alternatively, a $\chi^{2}$ can be constructed in terms of the differences in $F(Z)$ (see Eq. (1)) between any pairs of $n$ values and minimized as a function of $c$. These procedures yield essentially the same results. These results are reported in Fig. 2 for the ${ }^{129} \mathrm{Xe}+{ }^{197} \mathrm{Au}$ and ${ }^{36} \mathrm{Ar}+{ }^{197} \mathrm{Au}$ reactions.

It is interesting to notice that for all reactions and bombarding energies the quan- 


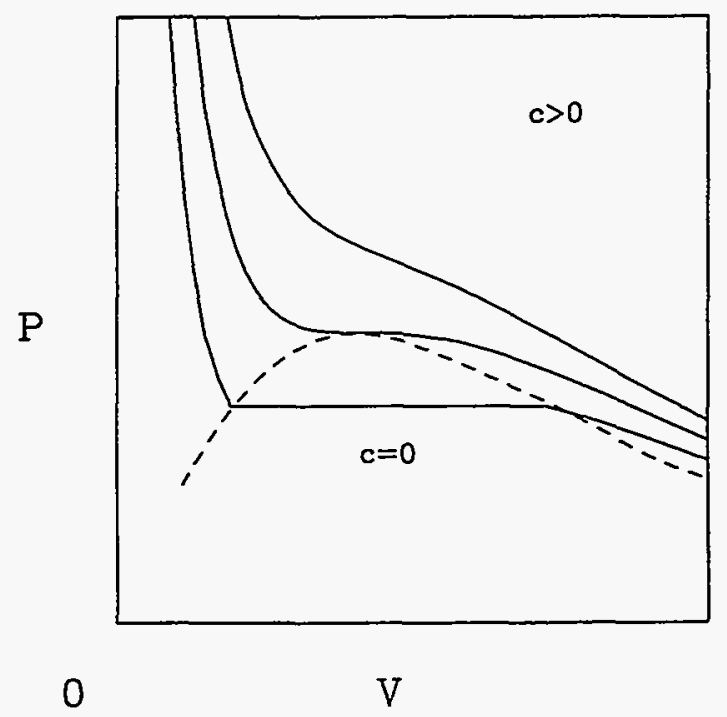

Fig. 3. Liquid-gas phase diagram. The top of the dashed line is the critical point. The area below the dashed line $(c=0)$ is the region where mixtures of the liquid and gas phases coexist.

tity $c$ starts at or near zero, it increases with increasing $E_{t}$ for small $E_{t}$ values, and seems to saturate to a constant value at large $E_{t}$.

This behavior can be compared to that of a fluid crossing from the region of liquidvapor coexistence to the region of overheated and unsaturated vapor (see Fig. 3). In the coexistence region, the properties of the saturated vapor cannot depend on the total mass of fluid. The presence of the liquid phase guarantees mass conservation at all average densities for any given temperature. A change in mean density (volume) merely changes the relative amount of the liquid and vapor, without altering the properties of the saturated vapor. Hence the vapor properties, and, in particular, the cluster size distributions cannot reflect the total mass or even the mean density of the system. In our notation, $c=0$.

On the other hand, in the region of unsaturated vapor, there is no liquid to insure mass conservation. Thus the vapor itself must take care of this conservation, at least grand canonically. In our notation, $c>0$.

This description should not be taken too literally, for a variety of reasons, one of which is the finiteness of the system. The $c=0$ regime may signify an evaporative-like emission from a source which survives as a charge conserving residue (liquid), while the $c>0$ regime may signify the complete vaporization of the source.

In order to test these ideas for a finite system, percolation calculations ${ }^{8}$ were performed for systems of $Z_{0}=97$ and 160 as a function of the bond breaking probability $p_{b}$. Values of $c$ were extracted (using Eq.(4)) as a function of $p_{b}$.

The results are shown in Fig. 4. For values of $p_{b}$ smaller than the critical (percolating) value ( $p_{b}^{\text {crit }} \approx 0.753$ for an infinite system), we find $c=0$. This is the region in which a large (percolating) cluster is present. As $p_{b}$ goes above its critical value, the 


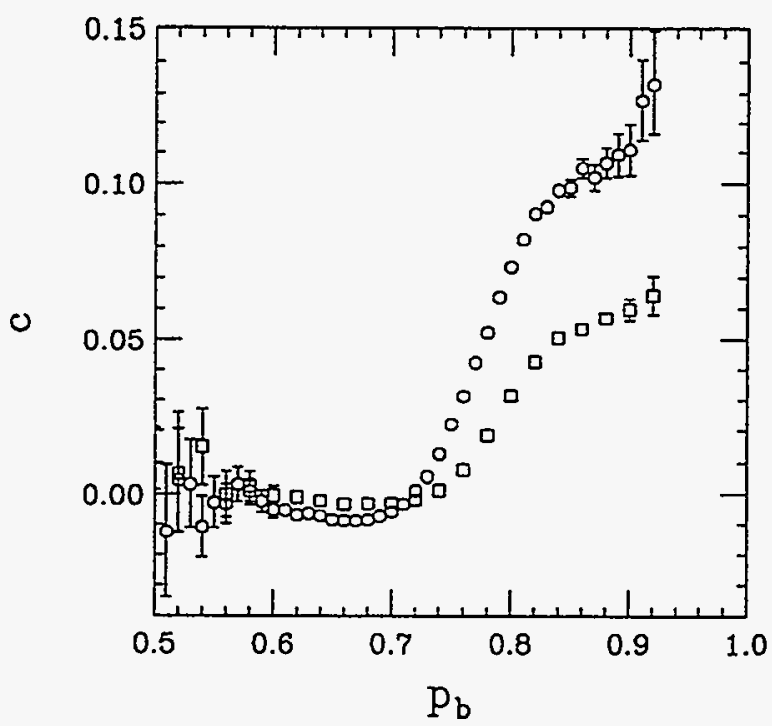

Fig. 4. A plot of $c$ versus the bond breaking probability $p_{b}$ from a percolation calculation ${ }^{8}$ for two systems $Z_{0}=97$ (circles) and $Z_{0}=160$ (squares). The statistical error bars are shown for errors larger than the symbol size.

value of $c$ increases, and eventually saturates in a way very similar to that observed experimentally. Due to the finiteness of the system the transition is smooth rather than sharp and can be made sharper by increasing the size of the system.

The significance of the actual experimental value of $c$ in the region where it seems to saturate is unclear. In Eq.(3), $c$ takes a direct meaning for the Euler problem: $c=1 / Z_{0}$. It should be noted that our analysis is not directly comparable to the Euler solution (Eq.(3)) since we have restricted ourselves to a limited region $(3 \leq Z \leq 20)$ of the total charge distribution for our study of how the source is partitioned into different IMF multiplicities. It must also be appreciated that Eq. (3) and the associated dependence of $c$ upon $Z_{0}$ are characteristic of a one-dimensional percolation model. In light of the points mentioned above, it is not unexpected that $c$ appears to be proportional, but not equal, to $1 / Z_{0}$ in the three-dimensional percolation calculation reported in Fig. 4. An interpretation of $c$ in terms of the source size may be possible when more data and a better understanding of the percolation of finite systems are available.

\section{Conclusions}

1) We have presented extensive evidence for an $n$ dependence of charge distributions of the form given by Eq.(1).

2) We have shown that the parameter $c$ increases from near zero at low $E_{t}$ (excitation energy) to a saturation value at high $E_{t}$.

3) Using the analogy of liquid-vapor equilibrium we have argued that $c=0$ 
indicates the presence of two phases (liquid-vapor) while $c>0$ indicates the presence of one phase (unsaturated vapor).

4) We have shown that a percolation calculation carried out for finite systems can be analyzed in the same way as the data, and portrays the same dependence for $c$ as one moves from the region where a percolating cluster is present to one where such a cluster is absent.

\section{Acknowledgements}

This work was supported by the Director, Office of Energy Research, Office of High Energy and Nuclear Physics, Nuclear Physics Division of the US Department of Energy, under contract DE-AC03-76SF00098 and by the National Science Foundation under Grant Nos. PHY-8913815, PHY-90117077, and PHY-9214992.

\section{References}

1. M. E. Fischer, Rep. Prog. Phys. 67, 615 (1967).

2. G. Sauer, H. Chandra, and U. Mosel, Nucl. Phys. A264, 221 (1976).

3. G. Bertsch and P. J. Siemens, Phys. Lett. B 126, 9 (1983).

4. P. J. Siemens, Nature 305, 410 (1983).

5. H. Schulz, D. N. Voskresensky, and J. Bondorf, Phys. Lett. B 133, 141 (1983).

6. A. L. Goodman, J. I. Kapusta, and A. Z. Mekjian, Phys. Rev. C 30, 851 (1984).

7. J. Hüfner, Phys. Rep. 125, 129 (1985).

8. W. Bauer, Phys. Rev. C 38, 1297 (1988).

9. X. Campi, Phys. Lett. B 208, 351 (19S8).

10. J.E. Finn et al., Phys. Rev. Lett. 49, 1321 (1982).

11. W.G. Lynch, Ann. Rev. Nucl. Part. Sci 37, 493 (1987).

12. T. Li et al., Phys. Rev. Lett. 70, 1924 (1993).

13. W. Trautmann, U. Milkau, U. Lynen, and J. Pochodzalla, Z. Phys. A 344, 447 (1993).

14. M. L. Gilkes et al., Phys. Rev. Lett. 73, 1590 (1994).

15. L.G. Moretto et al., Phys. Rev. Lett. 74, 1530 (1995).

16. K. Tso et al., Lawrence Berkeley Laboratory LBL-36858 (1995).

17. L. Phair et al., Lawrence Berkeley Laboratory LBL-36730 (1995).

18. J.A. Lopez and J. Randrup, Nucl. Phys. A 503, 183 (1989).

19. J.A. Lopez and J. Randrup, Nucl. Phys. A 512, 345 (1990). 